\title{
VALORES DE UNA CIENCIA IMPURA ${ }^{1}$
}

\author{
Marta I. González García \\ Departamento de Ciencia, Tecnología y Sociedad. Instituto de Filosofía. CSIC
}

ABSTRACT This paper argues for the role of contextual values in the shaping of scientific theories and practices through decisions concerning the definition of research problems, methods and aims. The analysis of the development of sexology from its origins in 19 th century Germany to the feminist criticisms of Masters and Johnson's paradigm shows how contextual values, by setting epistemic priorities, contribute to the establishment of criteria for the acceptance or rejection of theories and practices.

KEY WORDS Contextual and constitutive values. Epistemic priorities. Sexology.
RESUMEN Este artículo defiende la participación de valores contextuales en el modelado de contenidos y prácticas científicas a través de decisiones relativas a los problemas, métodos y fines de la investigación. Analizando el desarrollo de la sexologia desde sus origenes en la Alemania del siglo XIX hasta las críticas feministas al paradigma impuesto por Masters y Johnson se mostrará el modo en que valores contextuales marcan las prioridades epistémicas estableciendo así los criterios de aceptación o rechazo de teorías y prácticas.

PALABRAS CLAVE Valores contextuales y constitutivos. Prioridades epistémicas. Sexología.

\section{Ciencia, tecnología y valores}

El campo disciplinar CTS (ciencia, tecnología y sociedad) está compuesto por una gran diversidad de programas de investigación, educación y gestión cuyo nexo común consiste en ocuparse de las interrelaciones entre la ciencia, la tecnología y la sociedad. En el campo de la investigación se trata de desenredar los a menudo invisibles hilos que entrelazan los tres ámbitos; en el campo de la educación, de formar ciudadanos (sean o no científicos y tecnólogos) conscientes y responsables ante estas complejas relaciones; y en el campo de la gestión, de promover políticas públicas de la ciencia y la tecnología democráticas y socialmente sensibles. Dentro de este panorama general, mi propuesta se encuadraría en el primer ámbito, el que trata de desentrañar los complejos modos en los que ciencia, tecnología y sociedad interactúan. Aún es posible delimitar algo más el foco de interés. La investigación CTS se divide a menudo en dos tradiciones o enfoques diferenciados según la dirección de las relaciones ciencia-tecnologíasociedad que enfaticen (González García, López Cerezo y Luján López, 1996). Por una parte, la tradición académica (o europea) se ha ocupado especialmente de analizar el modo en que la sociedad condiciona el desarrollo científico-tecnológico; mientras que la tradición activista (o norteamericana) se ha centrado en las consecuencias de ese desarrollo sobre nuestras formas de vida. Se trata ésta de una diferenciación que responde a distintos origenes, disciplinas básicas y objetivos, y que a menudo origina controversias de difícil solución. No obstante, un tratamiento adecuado de las relaciones cienciatecnología-sociedad ha de recorrer necesariamente este doble camino: de la sociedad a la ciencia-tecnologia y de éstas de nuevo a la sociedad, porque compatibilizar las dos tradiciones CTS es la única manera de dotar de sentido a los esfuerzos en los ámbitos educativo y político.

En cualquier caso, esta contribución puede describirse como perteneciente a la tradición académica de analizar cómo factores sociales contribuyen al modelado de teorias y prácticas científico-tecnológicas. De hecho, en ella se defiende la participación de valores contextuales en el contenido y la práctica científica, y se ilustra esta participación con un caso de estudio: la investigación científica sobre la conducta sexual humana. Sin embargo, me gustaría insistir en que la tesis que se defiende y el tratamiento del caso de estudio intentan superar tanto el diálogo de sordos entre las dos tradiciones CTS como la mera acumulación de reconstrucciones sociológicas de episodios científicos (Shapin, 1982).

El caso de estudio que se analiza, la sexologia, presenta una historia cuya dinámica se mueve al ritmo de importantes cambios sociales al margen de los cuales su comprensión no resulta posible. Al mismo tiempo, sin embargo, las criticas más recientes al "paradigma" impuesto por Masters y Johnson (1966) reflejan la preocupación «activista» por las repercusiones sociales de una concepción reduccionista y normalizadora de la sexualidad, especialmente para las mujeres. 
Es éste un caso en el que se muestra con claridad la relación de doble dirección que mantienen la ciencia y la sociedad, asi como la insuficiencia de ocuparse de un único sentido de la misma.

Por otra parte, la discusión del caso se enmarca dentro del debate sobre ciencia y valores, uno de los escenarios centrales de la reflexión contemporánea sobre la ciencia y la tecnologia ${ }^{2}$. Tipicamente, cuando se habla sobre ciencia y valores se hace con dos tipos de propósitos. Por un lado se encuentran los intentos de demostrar que la ciencia supuestamente pura no es realmente tan pura, y que la participación de diversos tipos de valores no epistémicos en su construcción o justificación no la convierte automáticamente en "mala ciencia" (aquí incluiriamos la heterogénea e interdisciplinar literatura de la rama académica de CTS, o lo que se ha denominado estudios sociales de la ciencia). Por otro lado, resistiendo a los ataques, encontramos las posiciones de aquéllos que defienden que los únicos valores aceptables en ciencia son epistémicos, mientras que la intromisión de otro tipo de valores "externos" o contextuales debilita el estatuto epistemológico del producto resultante ${ }^{3}$. Ambas posiciones se ilustran con ciertos ejemplos de prácticas científicas. Las matemáticas o la física teórica se encuentran entre las ciencias aparentemente más puras y son, por tanto, importantes objetivos del análisis sociológico. La mejor forma de demostrar que la ciencia está inherentemente cargada de valores es analizar algunos de sus productos más indiscutibles. Las pseudociencias y los fraudes son, por otra parte, el tipo de casos preferidos de la perniciosa influencia de los valores externos en el desarrollo científico. Se recurre a ejemplos de mala ciencia, como el lysenkoísmo, la ciencia nazi o el más reciente caso de la fusión fría, para mostrar las nefastas consecuencias de la intromisión de los valores. La buena ciencia, por definición, no puede someterse a este tipo de análisis. Los valores contextuales son exclusivos de los productos descarriados del conocimiento científico.

Sin embargo, una característica insatisfactoria de muchos análisis del papel de los valores contextuales en las teorias y prácticas científicas es la poca atención que se presta habitualmente a los mecanismos por medio de los cuales intervienen. Sin el análisis de estos procesos, sin embargo, la identificación de carga valorativa en los productos cientificos (de sociedad en la ciencia) pierde fuerza argumentativa y queda sometida a la capacidad del narrador de contar historias en forma de reconstrucciones sociológicas tan inadecuadas y discutibles como las tradicionales reconstrucciones racionales a las que intentan sustituir.

Desde luego, numerosos autores han intentado dar cuenta de la presencia de valores contextuales: Barnes habla de intereses y objetivos, Collins recurre a los mecanismos retóricos de clausura de las controversias, Latour al establecimiento de alianzas... En todo caso, parece tratarse fundamentalmente de técnicas persuasivas las que concurren para traducir en productos científicos determinadas inquietudes extracientíficas. Esta visión parece implicar la idea intuitiva de cierta intencionalidad por parte de los científicos en su interés por promover determinadas ideologías, intereses profesionales o personales..., que en la mayor parte de las ocasiones no puede considerarse el caso. Una propuesta más interesante es la de Helen Longino (1990), según la cual las relaciones evidenciales entre observaciones e hipótesis se consideran mediadas por compromisos teóricos previos que dependen del contexto en el que los datos son evaluados. Diferentes "presuposiciones implicitas" (background assumptions), condicionadas por determinados valores contextuales, conducirán a interpretaciones distintas de los datos obtenidos experimentalmente. Por su parte, López Cerezo y Luján López (1989) han propuesto que la actuación de factores sociales se produce mediante su contribución a la selección de las prácticas metodológicas, incluyendo principios generales (como la identificación y definición de los problemas a solucionar y el enfoque básico adoptado) y prácticas metodológicas específicas (como modelos estadisticos o diseños experimentales).

Estas propuestas, además de subrayar la importancia de atender a la dimensión práctica de la ciencia para dar cuenta de la influencia de factores sociales, apuntan a la compleja interacción entre los valores contextuales y los valores considerados internos a la actividad científica. Los valores contextuales participan en el modelado de contenidos y prácticas científicas a través de decisiones relativas a los problemas, fines y métodos de la investigación. Estas decisiones, normalmente adscritas al "contexto de descubrimienton y consideradas irrelevantes para la evaluación de los productos científicos, muestran ser, por el contrario, centrales para los procesos de justificación, ya que los criterios de aceptación o rechazo son en cierta medida dependientes de las prioridades epistémicas cuyo establecimiento ha estado mediado por valores contextuales. El contexto de justificación no puede, entonces, independizarse completamente del contexto de descubrimiento en la medida en que la

ARBOR CLXXXI 716 NOVIEMBRE-DICIEMBRE (2005) 501-514 ISSN: 0210-1963 
evaluación de teorías (su aceptación, crítica o rechazo) dependa de aquellas prioridades epistémicas contextualmente seleccionadas. Analizar, entonces, estadios de ciencia en los que aún no se ha producido la estabilización de un sistema teórico-práctico (contexto de descubrimiento) 0 momentos de critica a sistemas más o menos estabilizados (contexto de justificación) parece una estrategia más apropiada para explorar el carácter contextual de la ciencia (es decir, la influencia de la sociedad) que la mera búsqueda de analogias o afinidades entre valores sociales y productos científicos.

Estas son las razones de la elección de un caso de estudio, la investigación sobre el comportamiento sexual, descarnadamente "impuro". "Impuro" por presentar una importante carga valorativa ligada a conflictivas preconcepciones sociales, lo que ha dificultado a lo largo de la historia su estabilización en torno a un cuerpo teórico y práctico no problemático. No creo, sin embargo, que estas caracteristicas puedan considerarse debilidades del caso de estudio escogido que resten fuerza a la argumentación general que intenta ilustrar ${ }^{4}$. Más bien al contrario, al mostrar la ambigüedad de lo que cuenta como valor "interno" y "externo" en estadios de ciencia preparadigmatica, este ejemplo permite ensayar la hipótesis de que ciencia completamente "epistémica" es imposible de construir y, del mismo modo, que es imposible evaluar de un modo estrictamente "epistémicon desarrollos científicos poco consolidados.

La primera parte de esta afirmación no es problemática: nadie negaria el papel crucial que cumplen fines, valores o preocupaciones de carácter no epistémico a la hora de definir y formular problemas interesantes para la investigación científica. Es en el caso de la evaluación de teorias e hipótesis, sin embargo, donde el papel de los valores se problematiza. En una ciencia que se encuentra en el proceso de negociar lo que pueden considerarse formulaciones de problemas, prácticas y teorias propias, tal evaluación de sus procesos y productos embrionarios se muestra de una forma muy clara ligada al tipo de fines, expectativas y preconcepciones asociadas a su origen en primer lugar; y es, de este modo, dependiente de valores tradicionalmente considerados "extracientíficos" (o únicamente aceptables en el "contexto de descubrimiento" en una práctica científica adecuada). A medida que la ciencia se desarrolla, y se asientan y consolidan prácticas y teorias, sin embargo, la distinción se va volviendo cada vez más clara hasta desaparecer la ambigüedad inicial y volverse ante nuestros ojos como obvia en si misma. Los estándares de evaluación se convierten, así, en rigurosamente "internos».

Existen casos, sin embargo, como el que nos ocupa, de ciencias especialmente problemáticas o «impuras» que continúan debatiendo la adecuación de problemas, fines, prácticas y teorias, pese a haber experimentado una considerable sofisticación teórica y metodológica. Más que tratarse de ciencias de algún modo «inferiores" y, por tanto, incapaces de alcanzar el grado de "desarrollo" y cristalización de las ciencias "duras", defendería que los interminables debates de carácter metodológico que se dan en ellas reflejan más bien la dificultad en llegar a un acuerdo acerca del tipo de valores epistémicos que priorizar, lo que, en última instancia, refleja desacuerdos básicos en valores contextuales en la comunidad de científicos involucrados. Suele tratarse de ciencias, como la sexología, pero también la psicologia, por ejemplo, que se ocupan de fenómenos especialmente uinteresantes» (tomando la expresión de S.J. Gould) no sólo para el científico y su comunidad en sentido restringido, sino para la sociedad en sentido amplio, y que, por tanto, se negocian en ese contexto más general, un contexto en el que llegar a acuerdos es mucho más trabajoso.

La sexologia nos proporciona uno de estos ejemplos en su estado más puro o, mejor dicho, más «impuro». Se trata de una ciencia emergente y sujeta a encendidas controversias en las que puede observarse de cerca la dinámica del uso de los distintos tipos de valores por parte de los propios cientificos al construir, justificar o criticar determinados desarrollos, en función de los fines percibidos de la investigación y de determinadas preconcepciones y expectativas. La sexologia es, efectivamente una ciencia abiertamente cart gada de valores contextuales, pero sin la participación de estos valores, referidos a cambiantes preconcepciones sociales sobre la naturaleza del comportamiento sexual y a las necesidades profesionales de los investigadores involucrados, no podría existir ninguna ciencia de la conducta sexual.

Narraré la historia del estudio científico de la sexualidad en tres tiempos: un primer periodo "preparadigmático" que se extiende hasta casi la segunda mitad del siglo XX; un segundo momento caracterizado por una mayor consolidación de la sexologia gracias al trabajo de Kinsey y de Masters y Johnson; y, por último, la crisis actual marcada por las criticas feministas al enfoque médico. Diferentes concepciones de la naturaleza y fines de la sexologia, guiadas por valores contextuales, contribuyen al establecimiento en cada 
una de estas etapas de distintos métodos de estudio y estándares de evaluación en la disciplina.

\section{El nacimiento de la sexología}

Como ocurre con un buen número de disciplinas modernas, podriamos aplicar a la sexologia la célebre frase que Ebbinghaus dedicó a la psicología: se trata de una disciplina con un largo pasado y una corta historia ${ }^{5}$. El largo pasado puede rastrearse hasta los griegos, en especial, hasta los escritos de Aristóteles sobre reproducción sexual. La corta historia comienza entre finales del siglo XIX y principios del XX, cuando el médico alemán Iwan Bloch acuña el término "sexología" o "ciencia sexual" (Sexualwissenschaft) y publica La vida sexual de nuestros tiempos y sus relaciones con la cultura moderna (1907), donde aboga por la cooperación de la medicina y las ciencias sociales en el estudio de la sexualidad humana. El único mérito de Bloch para pasar a la historia como iniciador de la sexología moderna no es el de haber dado por primera vez ese nombre a la disciplina. Bloch ofreció en su obra tanto un completo panorama del conocimiento sobre sexo en su época como detallados análisis de temas como la prostitución, la homosexualidad o las perversiones (que entendia, frente a la mayoria, como producto de "condiciones accidentales externas»). A la estela del trabajo pionero de Bloch, se produce otra contribución al establecimiento de la sexología como disciplina independiente cuando Magnus Hirschfeld crea en 1908 de la primera revista dedicada al estudio científico del sexo (Zeitschrift für Sexualwissenschaft). Poco después aparecerán las primeras asociaciones de sexologia: La Sociedad Médica de Sexología y Eugenesia (fundada por el propio Bloch en 1913) y la Sociedad Internacional para el Estudio del Sexo (fundada en el mismo año y también en Berlín por Albert Moll) ${ }^{6}$.

La historia del largo pasado, la que nos lleva de la Grecia de Aristóteles a la Alemania del cambio de siglo, es una oscura historia de observación mezclada con mito, prejuicios sobre el papel de los dos sexos y valoraciones morales. El conocimiento sobre anatomia genital, respuesta sexual y reproducción se desarrolla entretejido con ideas preconcebidas acerca de la naturaleza de los dos sexos ${ }^{7}$. Thomas Laqueur (1990) narra brillantemente el desarrollo de la problemática relación entre las ideas dominantes sobre los sexos y las investigaciones sobre las funciones reproductoras y la fisiología de los órganos sexuales. Según Laqueur, en el siglo XVIII se produce un cambio radical en el modo de abordar el estudio de los cuerpos y las funciones sexuales. Hasta entonces, y desde los griegos, habria dominado el modelo de "sexo único", en el que hombres y mujeres son dos variedades de un solo sexo y la diferencia se establece de un modo jerárquico (la mujer es un hombre imperfecto). El paso de este modelo al modelo ilustrado de dos sexos, en el que hombres y mujeres son inconmensurables, opuestos, es relativamente independiente de los avances en anatomía y fisiologia, es decir, de la experiencia y la observación, y está ligado a transformaciones epistemológicas, sociales y políticas ${ }^{8}$. Ambos modelos tienen consecuencias diferentes a la hora de ocuparse de la conducta sexual. Un buen ejemplo es el de los avatares históricos del orgasmo femenino. Durante mucho tiempo, de acuerdo al modelo de «sexo únicon, fue un lugar común que las mujeres debían experimentar orgasmo para que la fecundación fuera posible, pese a lo que podia parecer evidencia contraria. Deseo, ovulación, placer y fecundación se consideraban inseparables. La mujer, como el hombre, en los textos de Galeno, Alberto Magno o Avicena, experimenta deseo, erección de sus órganos genitales y orgasmo con eyaculación de su semilla femenina (Laqueur, 1990: 88ss). Tras la generalización del modelo de los dos sexos a partir de la llustración, el placer sexual femenino se disocia de la ovulación y de la procreación, de tal modo que la posibilidad de concepción sin orgasmo hace común la idea de una naturaleza femenina pasiva y poco interesada por los placeres del sexo. La mujer no siente el irreprimible deseo del hombre, y más que disfrutar, sufre 0 , en el mejor de los casos, tolera el sexo como deber conyugal encaminado a la procreación. Los nuevos hallazgos sirvieron para proporcionar fundamento científico para algunas viejas convicciones sobre la subordinación del sexo femenino y para algunas otras nuevas y convenientes, como la de que la mujer es un simple receptáculo pasivo del apasionado y fértil torrente masculino.

Paralelamente a las investigaciones en anatomia y fisiologia de la sexualidad y la procreación, gran parte del discurso sobre la conducta sexual estuvo dominado por la tradición judeocristiana, según la cual el sexo era una cuestión moral más que científica, y el fin de ocuparse de la sexualidad no era el de adquirir conocimiento acerca de un fenómeno natural, sino el de sancionar o censurar determinados comportamientos que se consideraban a priori moralmente correctos o incorrectos. La indeseabilidad moral de ciertas conductas (masturbación, adulterio, o cualquier tipo de práctica distinta al coito heterosexual vaginal) se tendia a reforzar aduciendo su responsabilidad en el desarrollo de 
diversos tipos de patologias físicas y psíquicas. Médicos del siglo XVIII como John Brown, Théophile de Bordeu, S.A.D. Tissot o Benjamin Rush escribieron alertando sobre los peligros de la masturbación, del sexo excesivo o de prácticas "antinaturales» para la salud física y psíquica ${ }^{9}$.

La preconcepción básica durante siglos de disquisiciones acerca del sexo era, efectivamente, la convicción profunda de que había una única forma válida de interacción sexual: aquella que estaba encaminada a la procreación; y las discusiones cientificas sobre conducta sexual trataban de identificar las condiciones que la hacian posible en el contexto del coito heterosexual. En parte debido al tabú que siempre ha rodeado el estudio de la conducta sexual, y en parte por el arraigado convencimiento de la existencia de conductas adecuadas e inadecuadas en el ámbito sexual, las investigaciones del siglo XIX que se consideran el origen de la sexología moderna aparecen, entonces, como análisis de las conductas desviadas, incluso cuando se complementan con una comprensión histórica y antropológica (de comparación intercultural), como en el caso de Iwan Bloch ${ }^{10}$.

Durante las primeras décadas del siglo $X X_{1}$ las teorias freudianas sobre la sexualidad fueron dominantes, aunque en investigación empírica fuera mucho más relevante, por ejemplo, el trabajo de Magnus Hirschfeld y Havelock Ellis. El atractivo de las ideas freudianas consistió en que proporcionaban un sistema global y un método de terapia destinado a tratar a pacientes disfuncionales. Especialmente interesantes, una vez más, fueron las teorias de Freud sobre el orgasmo femenino. La superación del complejo de castración (la "envidia del pene») por parte de la mujer, según Freud, implicaba abandonar el inmaduro orgasmo clitoridiano (en el que el clítoris se asocia al pene inexistente) por el maduro y equilibrado orgasmo vaginal. La incapacidad para alcanzar orgasmos vaginales llenó de mujeres las consultas de los psicoanalistas; y el orgasmo, que hasta entonces había recibido una atención irregular, pasó a ser el problema central de la sexología a partir de los años 20 (O'Connell Davidson y Layder, 1994: 13). Lo sorprendente de las propuestas freudianas es que, aunque estaban aparentemente fundamentadas sobre bases biológicas, se oponian a ideas cientificas ya existentes sobre el orgasmo femenino. Freud uinventan el orgasmo vaginal obviando el acuerdo general que situaba en el clítoris el centro del placer femenino y consideraba la vagina como relativamente insensible. Pese a la falta de evidencia empirica, las ideas freudianas contribuyeron a perpetuar el rol pasivo y limitado de la mujer y promovieron una larga amnesia sobre cuestiones de fisiología de la respuesta sexual femenina que ya habian sido descritas anteriormente con relativa sofisticación.

\section{El "nuevo realismo" estadounidense y la sexología moderna}

Hasta el momento, este brevísimo repaso de la historia de la investigación sobre sexualidad humana nos lleva desde el enfoque "patológico" que insistia en denunciar conductas inmorales y nocivas para la salud, pasando por el enfoque "antropológico" que miraba a otras culturas para estudiar la variación en los comportamientos sexuales, hasta llegar al enfoque "médico" o "biologicista" que ya vemos apuntado en Freud, aunque con sus peculiaridades propias. De la primitiva sexologia prescriptiva llegamos a una sexología que pretende combinar las funciones descriptiva y prescriptiva mediante la investigación empírica sobre conductas y órganos sexuales, y la detección y tratamiento de disfunciones.

Este es el periodo de la historia de la sexología que nos interesa especialmente y que se consolida a partir de finales de los años 40 con el ambicioso proyecto descriptivo de Alfred Kinsey, cuyos antecedentes pueden buscarse en el trabajo empírico que figuras como Gilbert V. Hamilton, Katharine Bement Davis y Robert Latou Dickinson llevaron a cabo en los Estados Unidos de principios de siglo. Con sus cuestionarios sobre prácticas sexuales y sus investigaciones sobre los cambios fisiológicos que ocurren durante la actividad sexual, ellos fueron los pioneros de lo que Bullough (1994: cap. 4) denomina el "nuevo realismo" de la investigación sobre el sexo.

La tendencia general en esta nueva etapa de la investigación sexológica fue la de un mayor interés en describir las conductas sexuales de las personas consideradas «normalesi" (frente al énfasis en los comportamientos de individuos "desviados" de las épocas anteriores), y un menor interés en el juicio moral, aunque con Masters y Johnson se impondría el juicio "terapéuticon. La publicación del primer informe de Kinsey, en 1948, señala cambios radicales en las actitudes hacia el sexo dentro y fuera de la ciencia. Los estudios de Kinsey, Masters y Johnson y otros, asi como la aparición de la figura del "terapeuta sexual», marcan el origen de la sexologia contemporánea.

ARBOR CLXXXI 716 NOVIEMBRE-DICIEMBRE (2005) 501-514 ISSN: 0210-1963 
Mediante la realización de entrevistas sistemáticas diseñadas y ejecutadas con una cuidadosa metodología, Kinsey emprende la tarea de aplicar al problemático campo del sexo los métodos de la ciencia más neutral. Se trata de recogida de datos y extracción de conclusiones, sin los prejuicios y adjetivos morales que parecian inseparables de un tema tan comprometido. J.B.S. Haldane resumió así la actitud de Kinsey con motivo de su muerte: "Nosotros, los cientificos, creemos que es nuestro deber describir el mundo tal como es (...). Ciertamente, no podemos dejar de lado el comportamiento sexual ni en nuestra descripción del mundo ni en nuestra explicación del mismon (cit. en Pomeroy, 1972: 471).

Kinsey se convirtió (junto con Wilhelm Reich y Herbert Marcuse, aunque con un talante mucho más "americano") en una de las figuras clave de la urevolución sexual». Especialmente revolucionario y liberador fue su volumen sobre la mujer. Revolucionario por los datos sobre la elevada incidencia de relaciones pre y extramaritales, datos que escandalizaron a la sociedad americana de la época (curiosamente, en el volumen sobre sexualidad masculina, lo escandaloso fueron los datos sobre homosexualidad, y no sobre relaciones sexuales fuera del matrimonio). Liberador por sus conclusiones acerca del orgasmo femenino ${ }^{11}$. Frente a las extendidas ideas freudianas, el trabajo de Kinsey revalora el despreciado orgasmo clitoridiano y revela que algunas mujeres son, incluso, capaces de orgasmos múltiples. La hembra humana, del mismo modo que el macho humano, concluye Kinsey, es un "animal capaz de experimentar orgasmos". Las críticas despertadas en los sectores más conservadores de la sociedad americana hiceron que, tras el volumen sobre sexualidad femenina, Kinsey dejara de recibir financiación del CRPS (Comité de Investigación sobre Problemas del Sexo) y la Fundación Rockefeller.

Desde luego que Kinsey también recibió importantes y acertadas críticas a la metodología de sus entrevistas. Kinsey habia diseñado cuidadosos mecanismos para evitar las mentiras y para extraer información de los sujetos más reticentes. Sin embargo, su sistema de muestreo era muy cuestionable. Las 5.940 entrevistadas y los 5.300 entrevistados que constituyeron la muestra para sus volúmenes sobre sexualidad femenina y masculina ${ }^{12}$ fueron todos ellos volunta-

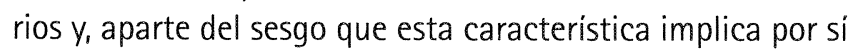
misma (podia tratarse de individuos especialmente deshinbidos en lo concerniente al sexo), la muestra presentaba diversos desequilibrios: habia demasiados sujetos del medio oeste de los EE.UU. (especialmente de Indiana), demasiados presidiarios entre los sujetos masculinos, demasiados blancos, demasiada gente culta, demasiados jóvenes... Difícilmente podría considerarse una muestra representativa.

La pretendida neutralidad de las entrevistas y los informes Kinsey implica, también algunas presuposiones debatibles y debatidas previas a las justificadas criticas metodológicas. Kinsey asumió una actitud decididamente "pro-sexo». Esto significaba la implícita aceptación de dos axiomas fundamentales: el sexo es bueno y cuanto más se practique, mejor (Irvine, 1990: 37). Pero también implicaba la renuncia al juicio moral y la aceptación como natural y apropiada de cualquier conducta sexual de la que los sujetos le informaran (cuyo rango podia variar desde ciertas preferencias ciertamente peculiares pero inocuas hasta la pedofilia). La renuncia programática a mezclar valores con hechós colocó a Kinsey y sus seguidores ante algunos dilemas morales dificiles de evitar. Consistemente, Kinsey se opuso con vigor a las leyes que regulaban las prácticas sexuales (que en algunos estados prohibian cualquier cosa que no fuera el coito heterosexual vaginal), señalando con acierto que sus datos mostraban que era tal el porcentaje de la población de ambos sexos que las violaba sistemáticamente, que resultaban claramente injustificadas, además de la imposiblidad de asegurar su cumplimiento. Junto a esto, y también de un modo coherente, Kinsey, cientificamente interesado en la respuesta sexual infantil, coleccionaba entrevistas con pedófilos, pero sin denunciarlos a las autoridades ${ }^{13}$. Este es un problema que sigue enfrentando el estudio científico de la sexualidad que pretende ser neutral y objetivo y no mezclar la "ciencia del sexo" con la "política del sexo", tarea que se revela repetidamente como condenada al fracaso. Esto sucede de un modo explícito, por ejemplo, cuando diferentes grupos de interés acuden al sexólogo como experto para apoyar posturas enfrentadas relativas a la pornografia, la prostitución o la homosexualidad.

Así y todo, uno de sus logros más importantes, y por el que ha pasado a la historia como un hito para la liberación de la mujer, fue su tendencia a afirmar que la sexualidad masculina y la femenina no son opuestas, como se habia venido sosteniendo desde el siglo XVIII, sino básicamente iguales.

Kinsey contribuye a un nuevo cambio en el modelo dominante sobre la naturaleza de los sexos. Como ya hemos mencionado, el modelo según el cual hombres y mujeres son 
versiones de una naturaleza sexual que es única habia sido generalmente aceptado durante la antigüedad y el renacimiento; los órganos genitales y la respuesta sexual en la mujer se consideraban análogos a los del hombre ${ }^{14}$, y por tanto se creía que, lo mismo que no podía haber fecundación sin eyaculación y orgasmo masculino, también el orgasmo femenino (con su correspondiente eyaculación) era imprescindible para la fecundación. En la Ilustración, sin embargo, se impone el modelo de sexos opuestos e inconmensurables que la sexología moderna comenzaba a poner ahora en tela de juicio. Del mismo modo que las investigaciones sobre fisiología de los órganos sexuales y los procesos de reproducción no son suficientes para explicar el abandono del modelo de sexo único en el siglo XVIII, el nuevo cambio apuntado en el trabajo empírico y descriptivo de Kinsey sobre los comportamientos sexuales de los norteamericanos en los años 40 y 50 , de vuelta al modelo de sexo único, tampoco está determinado por los propios datos que Kinsey proporcionó.

El periodo que siguió a la segunda guerra mundial en Estados Unidos fue una época crítica en la que se estaban gestando las transformaciones en los roles de género que se generalizarian en los años 60 y 70 . La guerra había propiciado una mayor implicación de las mujeres en la fuerza laboral y la vida pública, pero la posguerra amenazaba con devolverlas a sus hogares y a sus roles pasivos. El valor de la vida familiar y el papel de la mujer como esposa y madre abnegada, más interesada por las labores domésticas que por los placeres del sexo, constituyeron la imagen pública, pero hipócrita, de una sociedad que tras este telón publicitario escondia las sorpresas desveladas por Kinsey. La mecha encendida por los informes Kinsey acabaría de detonar en las décadas siguientes.

Los informes publicados por Kinsey promovieron, efectivamente, el tratamiento de la sexualidad femenina en el mismo plano que la masculina, pero aún enfatizaban ciertas diferencias básicas, como el hecho natural y no socialmente condicionado, según Kinsey, de que las mujeres tuvieran menos interés por el sexo y que algunas de ellas ni siquiera estuvieran "equipadas" para experimentar placer $^{15}$. La actitud de Kinsey era esencialista y naturalista: "el sexo es una función biológica, aceptable en cualquier forma en que se manifieste». De esta manera, la manifestación diferencial de las sexualidades masculina y femenina es un fenómeno "natural" que debemos a la historia de nuestra especie y que, del mismo modo que la expresión de conductas sexua- les socialmente condenadas, no debería ser objeto de juicio 0 intento de modificación.

La vuelta al modelo del sexo único, sin embargo, es ya definitiva diez años más tarde, en el trabajo de William $\mathrm{H}$. Masters y Virginia Johnson (1966). Sus investigaciones sobre la dinámica fisiológica del sexo resultaron ser un complemento muy apropiado para el impresionante material proporcionado por las entrevistas de Kinsey. Masters y Johnson midieron los cambios fisiológicos que ocurrian durante la actividad sexual: la frecuencia cardiaca, la presión arterial, las contracciones musculares, la lubricación y dilatación vaginal... Se trataba de un paso más en el proceso de convertir el estudio del sexo en una tarea científica objetiva y respetable. Lo mismo que en el caso de Kinsey, tampoco Masters y Johnson contaron con una muestra de sujetos representativa, dado que ahora se trataba de sujetos dispuestos a practicar el sexo ante los investigadores y sus cámaras, con ló que la autoselección era aún más evidente que en el caso de los entrevistados de Kinsey ${ }^{16}$. Esto, sin embargo, no supuso ningún problema para ellos, puesto que uno de los supuestos básicos de su trabajo, sobre el que implicitamente se apoyan para hacerlo a continuación aparecer explícitamente, en una hábil maniobra, como resultado de la propia investigación (de hecho, como el resultado más duradero de su investigación), es que la fisiología básica de la respuesta sexual es similar en todos los seres humanos. El resultado de esta presuposición-conclusión fue el "ciclo de respuesta sexualı, común para hombres y mujeres y consistente en cuatro fases sucesivas: (1) excitación, (2) meseta, (3) orgasmo y (4) resolución. El "ciclo de respuesta sexual» pretende describir el patrón de desarrollo natural de una interacción sexual adecuada, entendiendo por una uinteracción sexual adecuada" aquella conducente al orgasmo en ambos miembros de la pareja, típicamente, mediante el coito heterosexual vaginal. Mientras que Kinsey estaba interesado en la amplia variación de comportamientos sexuales y hacia hincapié en la diversidad, Masters y Johnson buscaban los universales de la respuesta sexual humana.

"Si quieres averiguar qué es lo que pasa, obviamente tienes que trabajar con aquéllos a quienes les pasan ${ }^{17}$, asi defendian los investigadores la parcialidad de una muestra que pretendia ser representativa de toda la humanidad, aunque de lo que era más bien representativa era de aquella parte de la población susceptible de acudir al especialista para

ARBOR CLXXXI 716 NOVIEMBRE-DICIEMBRE (2005) 501-514 ISSN: 0210-1963 
someterse a un tratamiento para sus problemas sexuales. Efectivamente, el trabajo de Masters y Johnson no tenía únicamente un propósito descriptivo, sino también prescriptivo y terapéutico. De hecho, en el prefacio de su obra La respuesta sexual humana (1966), esgrimen los problemas de inadecuación sexual como la causa principal de fracaso matrimonial que justifica su dedicación al estudio científico de la sexualidad. El ciclo de respuesta sexual permitía definir trastornos caracteristicos de cada una de las fases, y una consecuencia natural de su trabajo fue la creación de la figura del terapeuta sexual, con el propósito de tratar estas disfunciones. El éxito de las intervenciones terapéuticas que se desarrollaron guiadas por estas investigaciones fisiológicas sobre el sexo fue extraordinario y no se puede menospreciar, aún cuando el campo de la terapia sexual fue también invadido por toda clase de oportunistas. El esfuerzo de Masters y Johnson parecia no haber servido solamente para librar a la mujer de los últimos vestigios de oscurantismo y prejuicio ligados a su sexualidad, también le proporcionaba instrumentos para superar problemas y desarrollar todo su potencial.

Las entrevistas de Kinsey y el trabajo de Masters y Johnson sobre la fisiología del sexo originaron una nueva imagen de la sexualidad femenina. La propia organización de La respuesta sexual humana, de Masters y Johnson, parece mostrar una preocupación especial por revalorizar la respuesta sexual de la mujer. En su libro, Masters y Johnson empiezan describiendo la respuesta sexual femenina, a la que dedican más de 140 páginas; la respuesta del hombre, que encontramos a continuación, apenas merece 50. En la época de la segunda ola del feminismo, del «movimiento de liberación de la mujern, Kinsey, Masters y Johnson y los desarrollos paralelos en anticoncepción proporcionaron a las mujeres un contexto apropiado para poder desarrollar su sexualidad de una forma que hasta entonces les habia sido negada.

Las mujeres recibieron de forma entusiasta la nueva sexología porque presentaba unos resultados que eran a la vez producto de nuevas presuposiciones que surgian en un contexto social cambiante. El igualador "ciclo de respuesta sexual" de Masters y Johnson borraba una diferencia que siempre se habia considerado básica entre hombres y mujeres: la diferencia entre la sexualidad femenina y la sexualidad masculina. "Las mujeres necesitan una razón para tener sexo", decia el personaje de Billy Cristal en la película Cowboys de ciudad, "los hombres sólo necesitan un lugar» ${ }^{18}$. rencia ya no aparecia como inevitable, sino más bien como un producto social. El potencial sexual de la mujer era igual o incluso mayor al del hombre, y a partir de ahora podía y debía manifestarse libremente.

Masters y Johnson parecen haber proporcionado el primer acuerdo paradigmático en el campo de la sexologia: un método de trabajo basado en la observación de conductas sexuales y la medida de respuestas fisiológicas en condiciones de laboratorio; una generalización incuestionada: el ciclo de respuesta sexual; un modelo "mecanicista» de la conducta sexual; un ejemplar sobre el que se basa todo el sistema: el coito heterosexual vaginal en el que ambos miembros de la pareja alcanzan el orgasmo; y todo un programa de tratamiento para las disfunciones que no se acomoden al guión prescrito. No obstante, el «ciclo de respuesta sexual" fue más una elaboración conveniente que un simple descubrimiento. Masters y Johnson tuvieron que ejercer una considerable "violencia" sobre sus datos para acomodarlos en un modelo de sexo único y en un patrón de respuesta sexual universal desarrollado en cuatro etapas. Será este proceso de normalización y universalización el que autores posteriores, especialmente desde un óptica feminista, criticarán por considerar reduccionista e inapropiado. El trasfondo histórico de la investigación sobre el sexo aquí expuesto, junto con las características de estas críticas nos servirán para observar el modo en el que valores contextuales condicionan las prioridades epistémicas y, por tanto, la evaluación de productos y prácticas científicas en el caso de la sexología.

\section{Prioridades epistémicas y valores contextuales}

Masters y Johnson, apoyados en Kinsey, convirtieron el sexo en un acto natural; es decir, en una «función biológica sencilla y universal que, sin ninguna preparación, deben experimentar, gozar y realizar todos los seres humanos aproximadamente de la misma maneran (Tiefer, 1995: 37). Esta definición parece tener poco que ver con el enfoque moralizante y culpabilizador de los primeros tiempos, y asi contribuir al desarrollo de una actitud más abierta y tolerante con respecto al sexo. Sin embargo, las críticas feministas más recientes insisten en demostrar que el sexo "no es un acto natural» (Tiefer, 1995) que se pueda reducir a sus manifestaciones fisiológicas, sino más bien un "concepto" con significados cambiantes ligados inevitablemente a circunstancias sociohistóricas determinadas. Las criticas feminis- 
tas a las debilidades epistémicas del "paradigma» de Masters y Johnson no pueden entenderse sin hacer referencia a esta actitud anti-esencialista y los valores contextuales asociados a ella.

La historia de esta ciencia "impura» puede interpretarse en términos de la dinámica de valores epistémicos y no epistémicos (o contextuales) sugerida por Elizabeth Anderson (1996), y que sigue en parte al análisis de Helen Logino (1990) del papel de las presuposiciones del trasfondo en el establecimiento de la relación evidencial entre datos y elaboraciones teóricas. Según Anderson, ambos tipos de valores no se enfrentan, sino que más bien cooperan en el desarrollo de teorias y en la evolución de las disciplinas científicas $^{19}$. El interés de insistir en la diferencia entre oposición y cooperación radica en que gran parte de las reacciones alarmistas ante el relativismo supuestamente implicado en la defensa de la participación de valores contextuales en la génesis y evaluación de teorias cientificas asumen que ambos tipos de valores son excluyentes: si defendemos que las elecciones en ciencia están motivadas por valores constitutivos, entonces no hay lugar para los valores del contexto. Si, por el contrario, argumentamos que es preciso apelar a valores contextuales, estamos resignándonos al dogmatismo, el engaño, o la conversión de la ciencia en un conjunto de buenas intenciones. Sin embargo, este problema sólo surge cuando entendemos el fin de la ciencia como la mera acumulación de "verdades» 20 . Por el contrario, entendida la actividad científica a la manera kuhniana, la ciencia trata de resolver problemas o responder a preguntas, y en esta tarea, el propio problema o pregunta planteados marcan unos criterios de significatividad cuya naturaleza no es meramente epistémica. En este trabajo en equipo, los valores contextuales establecerían los estándares de significatividad de una teoría y la evidencia empírica determinaría si la teoría cumple tales estándares. Es decir, los valores contextuales mediarian las decisiones acerca de qué es lo que debemos buscar (qué preguntas plantear, cuál es el fin de la investigación...) y cómo debemos hacerlo (qué métodos particulares y procedimientos generales favorecer); mientras que la evidencia empírica nos aseguraria que, en ciencia, uno no siempre puede obtener lo que quiere.

Lo que está defendiendo Anderson no es simplemente que los valores externos intervengan en el "contexto de descubrimienton, en el origen de un problema que ha de recibir tratamiento científico. Según Anderson, el juicio acerca de teorias cargadas de este modo de valoración no puede ser independiente de esos mismos valores que las motivaron. En la evaluación de una teoría científica no simplemente se trata de juzgar su «adecuación a los hechos», sino también su significatividad con respecto al fin previsto para la investigación y, de este modo, la evaluación es en cierta medida función de ciertas presuposiciones, intereses o fines de carácter "extracientífico" con las que los científicos se plantean los problemas y se enfrentan a ellos.

Reformulando la propuesta de Anderson, podriamos decir que los valores contextuales, al dictar los estándares de significatividad de la investigación, seleccionan ciertas prioridades epistémicas que constituyen el marco para la determinación de métodos y resultados aceptables. La evaluación (la aceptación o el rechazo) puede considerarse, de este modo, epistémica, en la medida en que en ella se apela a criterios como la adecuación empírica o a valores cognitivos del tipo de los propuestos por Kuhn (precisión, coherencia, amplitud, fecundidad, simplicidad...). Sin embargo, la atención prioritaria a algunos de estos valores frente a otros requiere apelar a los fines de la investigación y los criterios de significatividad $y_{1}$ así, a su carga contextual ${ }^{21}$. En este sentido podemos decir que se trata de prioridades epistémicas mediadas por valores contextuales.

Esta "mediación" contextual no se da únicamente en la génesis de las teorias, sino que cumple también un papel clave en la justificación, en la medida en que, como ya se ha señalado, la aceptación de una teoría es relativa a su significatividad. Especialmente interesantes son los casos de rechazo o de crítica de teorias. Criticar una teoría consiste a menudo en priorizar diferentes valores epistémicos sobre la base de cambios en determinados valores contextuales (González Garcia, 1999). En estos casos, como el que nos ocupa, la distinción entre crítica epistémica y crítica no epistémica se desestabiliza, como sucede en el caso de estudio nos ocupa.

\section{¿Qué más quieren las mujeres?}

La aparición del «nuevo realismo americano" y el nacimiento de la sexología moderna implican un cambio en los fines (y las presuposiciones asociadas) del estudio de la conducta sexual. Mientras que el fin de la primera época era el de la identificación y eliminación de la conducta sexual desviada (donde se definía como desviada toda aquella actividad sexual no procreativa, o uinmadura" según los criterios 
freudianos); en la nueva sexologia se trata de la descripción de la conducta sexual "funcional" (que ya no es la que resulta en la fecundación, sino la que conduce al orgasmo) y el tratamiento de la conducta disfuncional por parte de "expertos del sexon. Fueron los datos proporcionados por las entrevistas de Kinsey y las observaciones de Masters y Johnson, junto con el supuesto del carácter natural y universal de la experiencia sexual y el empeño en sustituir los juicios morales por juicios "cientificos", los que permitieron enunciar el patrón básico de actividad sexual funcional: "el ciclo de respuesta sexủalı, un patrón que se adecuaba perfectamente a los fines de la investigación y a un cambiante clima social en el que las mujeres conquistaban poco a poco parcelas de igualdad.

Sin embargo, muchas feministas no están satisfechas con la concepción de la sexualidad derivada de la investigación empirica y fisiológica. Autoras como L. Tiefer, M. Jackson, J. Irvine, C. Tavris o C. Wade, han señalado que la «igualdad sexual" representada paradigmáticamente en el "ciclo de respuesta sexualn de Masters y Johnson, no es en absoluto un modelo igualitario, sino una forma de conseguir que la experiencia sexual femenina se conforme a un patrón de normalidad que sigue siendo masculino. La sexología moderna ha pretendido mostrar que las mujeres tienen necesidades y deseos exactamente iguales a los de los hombres, sólo que un poco más lentos. En este sentido, no representaria un avance real frente al modelo de sexo único imperante hasta el siglo XVIII, según el cual la mujer era una versión imperfecta del hombre.

Las críticas feministas han puesto de manifiesto debilidades epistémicas del enfoque de Masters y Johnson que ponen en tela de juicio las pretensiones de universalidad de su ciclo de respuesta sexual. Han criticado la arbitrariedad de lá distinción de las cuatro etapas del ciclo (más tarde se admitiría una etapa más: la del deseo), que consideran una camisa de fuerza demasiado estrecha para sistematizar la complejidad de las reacciones y relaciones sexuales. Han llamado la atención sobre lo injustificado de insistir en la similaridad de la respuesta masculina y femenina, cuando ellos mismos reconocieron una mayor complejidad y variabilidad en las mujeres ${ }^{22}$. Y también han criticado la muestra sesgada de individuos y de prácticas sexuales que Masters y Johnson utilizaron para establecer el ciclo de respuesta sexual: matrimonios y algunos individuos solteros, todos ellos voluntarios de raza blanca y nivel sociocultural elevado, practicando el coito vaginal y la masturbación. Entre otras cosas, entonces, las feministas han rechazado las presuposiciones de que la respuesta sexual sea universal e innata; que consista en las reacciones de partes del cuerpo separables y separadas; que lo importante en el sexo sea el buen funcionamiento de los genitales; y que la actividad sexual "normal" consista en la penetración del pene en la vagina (las mujeres, y los hombres, que no alcanzan el orgasmo de este modo podian merecer el diagnóstico de "orgasmo inhibido" según el DSM-III ed. revisada,1987).

La tarea cientifica de reducir el sexo a sus elementos fisiológicos (a músculos, tejidos, arterias, nervios y "puntos mágicosin), el objetivo de gran parte de la sexología moderna, refleja una visión tradicionalmente masculina de la sexualidad, centrada en los genitales. La historia del "punto G" proporciona un buen ejemplo del empeño en igualar la sexualidad femenina a la masculina (Tavris, 1992). El punto $G$ representa, para muchos sexólogos, la reducción más absurda y extrema de la sexualidad femenina al patrón masculino, completada incluso con una "eyaculación» específicamente femenina 23 .

Lo que defienden las feministas es que el sexo no es un acto natural, sino histórica, social y psicológicamente definido, y que por tanto no se agota en sus aspectos biológicos y fisiológicos. Consecuentemente, la sexología debería reconocer e incluir en su agenda de investigación al menos dos aspectos que se encuentran ausentes:

- En primer lugar, lo que el propio Ernest Grafenberg (que presta su nombre al famoso "punto G») ya señaló en 1950 al afirmar que «no hay ninguna parte del cuerpo de la mujer que no proporcione respuesta sexualn. Es decir, las investigadoras feministas insisten en que el sexo ocurre en muchos lugares, empezando por el cerebro, y que se trata de reduccionismo inaceptable definir, por ejemplo, la excitación sexual en términos de lubricación vaginal si la mujer manifiesta no encontrarse sexualmente excitada, es decir, sin tener encuenta su experiencia subjetiva.

- En segundo lugar, que pese al loable esfuerzo de los sexólogos por igualar ambos sexos, en este momento y en esta sociedad podemos identificar demasiados factores externos imposibles de obviar y que contribuyen a que la experiencia sexual de la mujer sea muy distinta a la del hombre. Además de los más evidentes, como son la elevada incidencia de acosos, explotación, abusos y violáciones sexuales de diversos tipos; hay otros 
condicionantes más sutiles, como la persistencia del "doble estándar" (que enjuicia de modo diferencial la asertividad sexual en hombres y mujeres), los problemas de autoimagen y las dudas sobre el atractivo fisi$\mathrm{co}$, la preocupación del embarazo, el a menudo frustrante uso del sexo para paliar carencias afectivas...

"Las teorias de la sexualidad", afirma Carol Tavris (1992: 212) criticando el paradigma impuesto por Masters y Johnson, "cambian dependiendo de la perspectiva de los cientificos que las construyen. Las ideas modernas sobre la sexualidad están tan marcadas por los sesgos de su tiempo como las teorias victorianas a las que sustituyen; es simplemente que los sesgos difieren [...] Las teorias que explican la sexualidad casi por completo en términos de los genitales de los participantes son tan limitadas como aquellas que ignoran por completo los genitales de los participantes".

El razonamiento de Tavris es perfectamente válido, pero el mismo rasero debe aplicarse a su propio análisis. Es decir, sus ideas acerca de lo que debería ser una teoría adecuada de la sexualidad dependen también, por supuesto, de su perspectiva y del convencimiento de que una teoría tal debería abandonar el énfasis genital para tratar el cuerpo como un todo y ocuparse de los aspectos emocionales y sociales que rodean las manifestaciones sexuales. No se trata, entonces, de que la perspectiva feminista sea privilegiada o neutral en algún sentido absoluto. Al criticar la teoría de la sexualidad asumida y reflejada, por ejemplo, en los tratados de Masters y Johnson por estar "sesgada", lo que están haciendo no es decir que sea errónea en el sentido de que la evidencia contradiga la teoria, sino más bien, que la evidencia que Masters y Johnson recogieron no es la evidencia relevante para los nuevos fines y valores que ellas proponen. Podriamos decir entonces que "criticar", en este sentido, consiste en priorizar los valores epistémicos o cognitivos (en este caso, por ejemplo, la uheterogeneidad ontológican frente a la simplicidad ${ }^{24}$ ) que mejor armonicen con determinados fines y valores contextuales, lo que nos induce a recabar evidencia empírica de distinto signo.

Las diferentes reacciones provocadas por los informes publicados por Shere Hite en los años 70 y 80 reflejan las diferentes prioridades epistémicas defendidas desde los enfoques "médicos" y los enfoques que podriamos denominar "culturales" favorecidos por las feministas. Lejos de la distancia, la neutralidad y la objetividad tan insistentemente buscada por Kinsey y Masters y Johnson, los informes Hite consisten bási- camente en la narración de la experiencia subjetiva de los hombres y mujeres entrevistados. En el lado médico, las críticas han sido fundamentalmente metodológicas: un muestreo inadecuado que solamente podía resultar en generalizaciones erróneas (el hecho de que el informe sobre la mujer, por ejemplo, se haya hecho en base a cuestionarios enviados a organizaciones femeninas habria tenido como consecuencia la presencia de un número no representativo de mujeres que se quejaban amargamente de sus relaciones con los hombres ${ }^{25}$ ) y una actitud general que no se atiene a los estándares de cientificidad pretendidos en el campo. Son criticas consistentes con los valores epistémicos favorecidos en el enfoque médico: rigor metodológico y atención exclusiva a las reacciones fisiológicas con el fin de establecer las caracteristicas básicas y universales de la respuesta sexual humana.

Estos no eran, sin embargo, los fines y las prioridades de Hite. En consonancia con las criticas feministas, Hite estaba más interesada en las experiencias subjetivas de sus sujetos y en los significados sociales asociados a las prácticas sexuales $^{26}$. Hite no se considera una científica, ni siquiera una cientifica social, sino más bien una historiadora cultural. De acuerdo con el fin de documentar sentimientos y significados, Hite prefiere el método cualitativo de dejar que sus sujetos uhablen por si mismos». Los informes Hite apenas proporcionan datos nuevos sobre tipos o frecuencias de actos sexuales, pero sí acerca de un gran número de mujeres que informan de desajustes sexuales y emocionales provocados por presiones sociales o falta de entendimiento con los hombres (cuando no por diversos tipos de maltrato) ${ }^{27}$.

Los desacuerdos respecto al fin previsto de la investigación, que tienen una importante carga contextual, se traducen en distintas y opuestas prioridades epistémicas: en un lado se favorece la simplicidad para facilitar las aplicaciones terapéuticas y asegurar el rigor científico (ambos necesarios para lograr la respetabilidad de la disciplina), en el otro la complejidad para defender el compromiso social. El resultado es dos formas de hacer sexología que presentan un aspecto muy distinto y que se critican mutuamente esgrimiendo como arma sus propias prioridades epistémicas desdeñadas desde la posición rival.

\section{Consideraciones finales}

He defendido que no es posible evaluar la investigación sobre el sexo independientemente de fines y valores "externos". La

ARBOR CLXXXI 716 NOVIEMBRE-DICIEMBRE (2005) 501-514 ISSN: 0210-1963 
dominancia del enfoque médico en la sexología actual, sin embargo, no puede explicarse únicamente en términos del interés por acomodar la sexualidad femenina a los estándares masculinos, como pretenden algunas de sus criticas. Otros factores, ya mencionados, han jugado sin duda alguna un papel fundamental, como la necesidad de la sexología de presentarse como una ciencia objetiva y racional pese a lo uimpuron de su objeto de estudio, para lo que no hay estrategia mejor que hablar friamente de músculos, tejidos y arterias y nervios (precisamente lo que las feministas les reprochan).

Algunos de los problemas que arrastra la sexología, y que sostienen aún hoy la incertidumbre acerca de su identidad, están relacionados con las dificultades de su profesionalización. De hecho, a finales del siglo XIX, al mismo tiempo que otras disciplinas, como la psicología, luchaban en Estados Unidos por su estatuto como ciencias a través de complejos procesos de institucionalización, los investigadores interesados por el sexo no tenian ningún interés en la creación de una disciplina independiente. Seguian definiéndose a si mismos como ginecólogos, urólogos, biólogos, psicólogos o antropólogos (Bullough, 1994). Este desinterés por la independencia de la disciplina, producto de la reticencia de las instituciones académicas a librarse de los tabúes que rodean al sexo (aún en nuestros dias es recomendable que alguien interesado en la investigación sexológica se labre antes un sólido prestigio en alguna otra área menos controvertida), tuvo un papel básico en mantener a la sexología como una ciencia con una identidad incierta. La búsqueda de respetabilidad profesional y el interés por las aplicaciones terapéuticas que proporcionarian aceptación social son valores (contextuales) que han reforzado el enfoque médico criticado por las feministas por sus aspectos reduccionistas y androcéntricos.

En cualquier caso, las críticas feministas a los presupuestos y resultados del enfoque de investigación en sexología dominante, sin embargo, no se limitan a mostrarnos cómo esta investigación está condicionada y modelada por fines y valores "extracientificos". Cuando Sandra Harding (1989) expone las modalidades existentes de epistemologias feministas, comienza hablando de lo que ella denomina uempirismo feministan, que consiste en identificar sesgos sexistas en determinadas teorias cientificas. Continúa defendiendo que se trata de un tipo de epistemología "conservadora", en el sentido de que no cuestiona la posibilidad de una ciencia libre de valores externos. Sin embargo, la propia Harding no puede dejar de intuir el "futuro radical» del empirismo feminista. Un futu- ro radical que reside en la perplejidad que nos produce el hecho de que gran parte de los desarrollos científicos que las feministas critican de manera tan convincente son teorias que nos cuesta calificar de umala ciencia», o cuyos defectos sólo aparecen al contemplarlas desde un conjunto distinto de fines y valores. Es decir, el empirismo feminista no tiene más remedio que superarse a si mismo para defender que tal ciencia libre de valores es una quimera y que sus justificadas criticas epistémicas son inseparables de valores contextuales. De este modo, el empirismo feminista no puede limitarse a identificar el origen «impuro» de la investigación que critica; en el mismo movimiento queda también en evidencia la «impurezan (no debilitante) de su propia crítica. La evaluación de teorías no puede ser entonces independiente de los valores contextuales que originaron la investigación o dieron pie a la crítica, porque lo que está en juego no es únicamente la verdad o la adecuación empírica, sino también la significatividad, que es un criterio relativo a la pregunta o problema planteado y al fin de la investigación. De este modo, en la medida en que no puede separarse de sus orígenes "impuros", no hay nada que pueda hacer la sexología para dejar de ser una ciencia "impura".

Aún nos quedaría responder a la pregunta de qué puede enseñarnos el caso en tantos sentidos peculiar de la sexología acerca de la participación de valores contextuales en otras ciencias de apariencia menos «impuran. La similaridad de este episodio, por ejemplo, con otros en los que críticas feministas han identificado valores androcéntricos y propuesto alternativas (primatología, psicología, biología, paleoantropologia, arqueología...) parece indicar que la sexologia no es, después de todo, tan peculiar en su «impureza». Desde luego, no podemos considerar que los casos objeto de crítica feminista agoten la dimensión contextal de la ciencia. Disputas sobre los fines y valores epistémicos prioritarios contextualmente mediadas pueden encontrarse también de forma general en los estadios preparadigmáticos de diversas disciplinas cientificas. No obstante, atender al modo de funcionamiento de la crítica feminista en la ciencia, una critica desde la ciencia que tiene el objetivo explícito de impulsar compromisos políticos, resulta también revelador a la hora de superar la incomunicación entre las dos tradiciones de los estudios CTS. La crítica feminista es un ejemplo de cómo la preocupación por la cuestión de la ciencia en la sociedad (las consecuencias sociales del desarrollo científico-tecnológico) puede de hecho beneficiar al análisis de la cuestión de la sociedad en la ciencia (los condicionantes sociales del desarrollo científico-tecnológico). 
1 Este trabajo ha sido financiado por la CICYTCRS/JR; exp. 2903, 2004-2006). Versiones anteriores se presentaron en el $I V$ Encuentro Hispano-Mexicano de Filosofía: Ciencia y Valores (Madrid, noviembre 1997) y en el curso Ciencia, Tecnología y Valores: Reflexiones en visperas de un nuevo milenio (Santa Cruz de Tenerife, UIMP, abril 1999). Me gustaria agradecer a Javier Echeverría, Amparo Gómez, León Olivé y Eulalia Pérez Sedeño su interés y sus comentarios criticos.

2 Para una presentación de los términos del debate, véase Echeverria (1995: cap. 3).

3 Entiendo por valores contextuales, siguiendo a Longino (1990) $r$ aquéllos pertenecientes al contexto histórico y social en el que la ciencia se lleva a cabo.

4 Véase en Nickles (1986) una discusión del uso de casos de estudio como evidencia para propuestas en fiosofia de la ciencia, y una defensa de su papel "ilustrativo" (frente a la función de instancias confirmadoras o falsadoras).

5 Para la historia de la investigación científica sobre la sexualidad, véase Bullough (1994).

6 No existe, sin embargo, un acuerdo general acerca del nacimiento de la sexología moderna como el que encontramos, por ejemplo, en historia de la psicologia al designar 1879 (la fecha en la que Wundt estableció el primer laboratorio de psicologia experimental en Leipzig). Entre las causas de esta diferencia deberiamos señalar particularidades del desarrollo interno de la sexología a las que nos referiremos más adelante; pero considero también relevante el hecho de que no exista una comunidad consolidada de historiadores de la sexología, lo mismo que existe para la historia de otras disciplinas cientificas.

7 Véase, por ejemplo, Laqueur (1990), Russett (1989) o Tuana (1993).
8 Algunas de estas transformaciones fueron: "El crecimiento de la religión evangélica, la teoria politica de la llustración, el desarrollo de nuevos tipos de espacios públicos en el siglo XVIII, las ideas de Locke sobre el matrimonio como contrato, las drásticas posibilidades de cambio social abiertas por la Revolución francesa, el conservadurismo postrevolucionario, el feminismo subsiguiente a la Revolución, el sistema fabril con su reestructuración de la división sexual del trabajo, el crecimiento de una economía de libre mercado de servicios y mercancias, el nacimiento de las clases [...]" (Laqueur, 1990: 33). Véase también en Celia Amorós (1997) la difícil relación de las mujeres con la llustración: el mensaje universalista en el que se sienten incluidas convive con el discurso de la diferencia que las excluye.

9 Bullogh (1994: 21) argumenta que la atribución de tantos males al sexo no se debía únicamente a prejuicios morales, sino también a la extensión de la sifilis y otras enfermedades venéreas y el desconocimiento de sus mecanismos de transmisión. No obstante, la condena del sexo no procreativo y fuera del matrimonio sobre la base de sus riesgos físicos y psiquicos sobrevivió a la explicación cientifica de la adquisición y curso de la sifilis.

10 Importantes excepciones a esta norma pueden encontrarse en investigadores homosexuales y mujeres. Se trata de dos grupos que, por razones obvias, tenian un especial interés en desterrar prejuicios asociados al sexo. Karl Heinrich Ulrichs, Magnus Hirschfeld, Celia Mosher o Katherine Bement Davis son algunos de los nombres reseñables en este contexto.

11 Conclusiones que sólo eran sorprendentes en el contexto de la hegemonia freudiana, ya que el clitoris fue desde el siglo $X V I I$ y de un modo generalizado en los manuales médicos del XIX, el órgano principal del placer femenino (Laqueur, 1990).

12 En total, Kinsey y sus colaboradores recogieron unas 18.000 historias, aunque su sueño de coleccionista compulsivo habia sido el de llegar a las 100.000 (véase Pomeroy, 1972).
13 La entrevista más larga de la colección de Kinsey, de 17 horas de duración, fue de hecho, la de un pedófilo que afirmaba haber practicado el sexo con cerca de mil preadolescentes de ambos sexos, junto a otras asombrosas proezas sexuales (Bullough, 1994: 175).

14 Aunque de algún modo "debilitados" o "subdesarrollados", el patrón ideal era el masculino.

15 Una curiosa e injustifcada intepretación, si tenemos en cuenta que uno de los hallazgos más relevantes de las investigaciones de Kinsey indica importantes diferencias en actitudes y comportamientos sexules en función de la clase social, hallazgo que Kinsey nunca interpretó como indicador de diferentes «naturalezas sexuales" según la clase social.

16 De hecho, Masters y Johnson comienzan su investigación con una muestra de prostitutas, que más tarde rechazarán para seleccionar individuos no "profesionales", que eran exclusivamente de raza blanca y mayoritariamente de nivel sociocultural elevado, seleccionados por su capacidad de "actuar" en público y de verbalizar sus reacciones sexuales.

17 Citado en Irvine (1990: 83).

18 Citado en Lips (1993).

19 Véase también González García (1999).

20 Más aún, si la consideramos como la acumulación de "cualquier verdad", porque, como señala Anderson (1996), no cualquier conjunto de verdades sobre un fenómeno constituye una teoría adecuada del mismo. Muchas de las verdades que se pueden decir sobre algo pueden ser triviales, parciales o irrelevantes.

21 Sobre la inconclusividad de los valores cognitivos en situaciones de infradeterminación, véase González García, López Cerezo y Luján López (1996: cap. 3).

22 De hecho, Masters y Johnson (1966) presentan tres posibles variaciones en el ciclo de respuesta sexual femenina, frente al patrón único masculino. 
23 Esta "eyaculación femenina» presenta una asombrosa similitud con el modelo antiguo de sexo único, donde se creia indispensable para la procreación. $Y$ es también aducida por algunos psicoanalistas para apuntalar su tesis de que el orgasmo "maduro" en la mujer es el orgasmo vaginal.

24 Longino (1996) propone una lista alternativa de valores epistémicos (en el sentido de que se trata de virtudes deseables en una teoría o hipótesis cientifica y que guian su evaluación) que suelen ser utilizados por las feministas en defensa de objetivos políticos: adecuación empírica, novedad, heterogeneidad ontológica, mutualidad de interacción, aplicabilidad a necesidades humanas y difusión de poder. Priorizar la heterogeneidad ontológica frente a la simplicidad, por ejemplo, hace preferibles teorias que enfatizan la diversidad en lugar de la uniformidad.

25 Recordemos, no obstante, que el problema del muestreo es también aplicable tanto en el caso de Kinsey como en el de Masters y Johnson.

26 Hite, no obstante, es también criticada por algunas feministas por su tratamiento individualista de los problemas de la sexualidad femenina y su incapacidad para ubicarlos en un contexto social y político más amplio (Irvine, 1990: 157).

$27 \mathrm{El}$ informe sobre sexualidad masculina también recoge las presiones sociales que sufren los hombres y condicionan su vivencia de la sexualidad.

\section{REFERENCIAS}

Anderson, E. (1996), "Knowledge, Human Interests, and Objectivity in Feminist Epistemology", Philosophical Topics. 23: 27-58.

Bullough, V.L. (1994), Sex in the Bedroom: A History of Sex Research, Nueva York: Basic Books.

Echeverría, J. (1995), Filosofía de la ciencia, Madrid: Akal.

González García, M.I. (1999), "Convergencia y conflicto de valores: el caso de las diferencias sexuales en habilidades cognitivas", en: A. Ambrogi (ed.), La Naturalización de la Filosofía de la Ciencia, Palma de Mallorca: Universidad de las Islas Baleares.

González García, M.I., J.A. López Cerezo y J.L. Luján López (1996), Ciencia, tecnologia y sociedad: una introducción al estudio social de la ciencia y la tecnología, Madrid: Tecnos.

Harding, S. (1991), Whose Science? Whose Knowledge?, Cornell University Press.

Irvine, J.M. (1990), Disorders of Desire: Sex and Gender in Modern American Sexology, Philadelphia: Temple University Press.

Laqueur, T. (1990), La construcción del sexo: cuerpo y género desde los griegos hasta Freud, Madrid: Cátedra, 1994.

Lips, H.M. (1993), Sex and Gender: An Introduction, 2 ed., Mountain View, CA: Mayfield.

Longino, H.E. (1990), Science as Social Knowledge, Princeton: Princeton University Press.

Longino, H.E. (1996), "Cognitive and Non-Cognitive Values in Science», en: L.H. Nelson y J. Nelson (eds.), Feminism, Science, and the Philosophy of Science, Dordrecht: Kluwer, 1996.

López Cerezo, J.A. y J.L. Luján López (1989), El artefacto de la inteligencia, Barcelona: Anthropos.

Masters, W.H. y V.E Johnson (1966), Human Sexual Response, Boston: Little, Brown \& Co.

Nickles, T. (1986), "Remarks on the Use of History as Evidencen, Synthese 69: 253-266.

O'Connell Davidson, J. y D. Layder (1994), Methods, Sex and Madness, Londres: Routledge.
Pomeroy, W. B. (1972), Dr. Kinsey and the Institute for Sex Research, Nueva York: Harper \& Row.

Russett, C.E. (1989), Sexual Science: The Victorian Construction of Womanhood, Cambridge, MA: Harvard University Press.

Schiebinger, L. (1989), The Mind Has No Sex? Women in the Origins of Modern Science, Cambridge, MA: Harvard University Press.

Shapin, S. (1982) "History of Science and Its Sociological Reconstructions", History of Science 20: 157-211.

Tavris, C. (1992), The Mismeasure of Woman, Nueva York: Touchstone.

Tiefer, L. (1995), El sexo no es un acto natural y otros ensayos. Madrid: Talasa, 1996.

Tuana, N. (1993), The Less Noble Sex: Scientific, Religious, and Philosophical Conceptions of Woman's Nature, Bloomington: Indiana University Press.

ARBOR CLXXXI 716 NOVIEMBRE-DICIEMBRE (2005) 501-514 ISSN: 0210-1963 